\title{
Research on Application of OGSA-based Digital Campus
}

\author{
Zhang Shunli ${ }^{\mathrm{a}}$, Yin Qingshuang ${ }^{\mathrm{b}}$ \\ ${ }^{a, b}$ The Department of Computer Science and Engineering, Henan Institute of Engineering, Zhengzhou, China
}

\begin{abstract}
This paper briefly introduces the concept and characteristics of Grid technology and Open Grid System Architecture (OGSA). By analyzing the deficiencies existing in the present campus network and the comparative advantages of campus Grid, it presents and discusses the model and design of OGSA-based campus Grid. Building campus Grid is an important work in Grid gaining ground; therefore, the study on OGSA-based Campus Grid is of great significance in applying Grid computing to educational field.
\end{abstract}

Index Terms: Grid Computing; Digital Campus; Campus Grid; OGSA

(C) 2012 Published by MECS Publisher. Selection and/or peer review under responsibility of the International Conference on E-Business System and Education Technology

\section{Introduction}

With the rapid development of computer and information technology, digital education is being rapidly enhanced in universities. At present, most colleges have their own campus network and build digital campus. As the main support platform, campus network plays more and more an important role in all walks of university life, such as teaching, scientific research and management and so on. However, because of heterogeneity of the entire Internet from hardware to software and lack of uniform standards among different educational institutions, the education resources on the internet cannot be fully shared, which leads to a lack of extensive resource sharing and severely repetitive developments of learning resources.

The Grid technology is the latest development in information technology. It combines and integrates heterogeneous resources in the whole Internet to form a super-computer, with stronger computing ability and more comprehensive resource sharing. For the moment, the most influential Grid system architecture is Open Grid Services Architecture (OGSA).Applying Grid technology to build an OGSA-based digital campus will effectively resolve the problems mentioned in the previous paragraph, and greatly alter the status quo and promote the further development of the digital campus.

Corresponding author:

E-mail address: ${ }^{\mathrm{a}}$ zhang_slxx@126.com; ${ }^{\mathrm{b}}$ yinqsh@163.com 


\section{Grid Concept and Grid Architecture}

\subsection{The concept of Grid computing}

The word Grid is used by analogy with electric power Grid, which provides pervasive access to electricity and, like the computer and a small number of other advances, has had a dramatic impact on human capabilities and society [1]. Its goal is that we can use network services as handy as electricity. When we use electric equipment, the only thing we need to do is insert the plug into the corresponding socket, we do not need to know where the electricity comes from, by what power it is supplied, how the power is transmitted or how we need to manage them. For the common user, all these are transparent.

Grid computing is a term referring to the combination of computer resources from multiple administrative domains to reach a common goal [2]. As an emerging technology, Grid is constantly developing and changing. It was originally developed to enable resource sharing within scientific collaborations; however, it has been widely used on increasingly larger scales. In fact there is no formal Grid definition which is generally accepted. According to my own understanding, Grid is defined as follows:

Grid is a technology to enable the sharing of all resources on the Internet to achieve interoperability, including hardware, software and communication resources.

\subsection{The Characteristics of Grid}

Grid is an emerging network infrastructure, and has the additional capabilities:

\section{1) distribution and sharing}

Distribution is the physical characteristic of Grid, and it means Grid resources are distributed; Resource sharing is one of the purposes of Grid, and it is the software support to realize Grid's logical features. One major purpose of Grid is to eliminate the resource isolated island so as to completely share resources over the Internet [3].

\section{2) Dynamic and Heterogeneity}

Grid is a collection of various resources and services. For a Grid system, its service, resources and size are constantly changing while new Grid resources and services are added or deleted. Heterogeneity means Grid can connect various heterogeneous network systems and the most extensive education resources [4].

\section{3) Supercomputing performance and Strong support services}

The Grid system can combine all computers together to become a virtual supercomputer [3]. Virtual laboratory, space and Tele-immersion network education services all need strong calculating ability, which is very difficult to realize in the traditional network environment, while being easily obtained in the Grid environment.

Grid can provide accurate service intelligently and quickly, according to the needs of users. When users input their own requests in Grid systems, the resources needed can be located and returned to them quickly and accurately.

\section{4) Self-similarity}

Self-similarity is a very common phenomenon in the natural world. It reflects the natural attributes of the world to a certain extent. Grid's self-similarity is explained that Grid structure is in accord with nature, or Grid to a certain degree is much closer to the laws of nature [4]. 


\subsection{Grid Architecture}

Grid architecture identifies fundamental system components, specifies the purpose and function of these components, and indicates how these components interact with one another [1]. For the moment, there are two main Grid architectures: LGA (Layered Grid Architecture) and OGSA (Open Grid Services Architecture). OGSA is the most influential Grid architecture. Here I just refer to OGSA.

OGSA is a service-oriented concept. A fundamental OGSA concept is that of the Grid service: a Web service that implements standard interfaces, behaviors, and conventions that collectively allow for services that can be transient (i.e., can be created and destroyed) and stateful( i.e., we can distinguish one service instance from another) [1].

OGSA is based on OGSI (Open Grid Services Infrastructure), OGSI defines a set of building blocks that can implement a variety of resource layer and collective layer interfaces and behaviors [1]. From the angle of Grid development to reflect Grid hierarchy, OGSA can be abstracted as four layered components shown in Fig.1, in sequence from bottom to top, they are: resource layer, service layer, application layer. In the Grid layered structure, all levels provide support upward for above layers, and proposes the corresponding all sorts of functions and means downward for the below layers.

\section{The Significance of OGSA-Based Digital Campus Construction}

The essence of digital campus construction is the use in schools as the information means to achieve the effective integration, distribution and utilization of various sharing resources, and realize the optimization and coordination of process of education and school management, which can vastly improve efficiency.

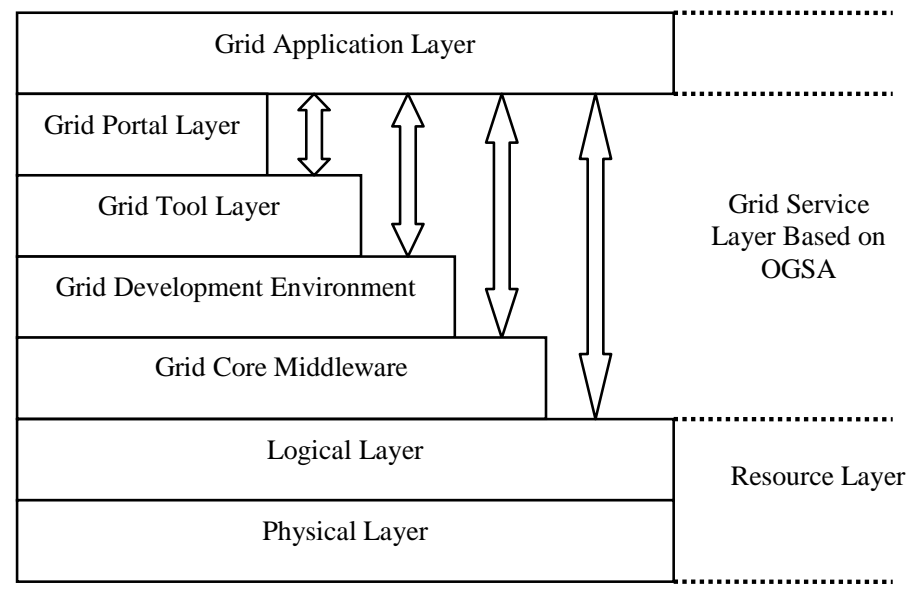

Fig. 1. The layered structure of OGSA

\subsection{Deficiencies in current digital campus networks}

The most important application of the digital campus in education is the sharing of resources and collaboration, however, the source sharing in current campus network systems are at relatively low levels, which are reflected in the following four areas: 
- Teaching resources are not unified in format, thus very difficult to undertake unified effective coordination [5].

- From the point of view of hardware and operating systems, because various heterogeneous systems coexist, application systems cannot run in different operating platforms.

- In terms of realization way, because most Web pages are static or dynamic, they don't reflect service concept [6].

- As for users, because campus network can't fully realize sharing information and services, they are still unable to obtain the most efficient and convenient services. What's more, such a structure creates a disadvantage on intercollegiate sharing and collaboration.

\subsection{The significance of campus Grid based on OGSA}

Applying OGSA to construct a digital campus can eliminate the deficiencies in current campus networks and achieve a higher level of sharing and collaboration [7], which are of great significance. The advantages are mainly reflected in the following three areas:

\section{1) Powerful Resources Management and effecitive Resource sharing}

Resource management and resource sharing is the core constructing of the digital campus [4]. Campus Grid based on OGSA possesses powerful resource management and efficient resource sharing to optimize resource allocation and adapt the system to the various needs of users. At the same time, it ensures users can make full use of all idle resources thereby improving the efficiency of resource utilization.

\section{2 ) Transparency and Intelligence Services}

OGSA is service-oriented Grid architecture [3]. It can provide personalized service at a higher level for users, which allow access to the resources they need in the shortest possible time.

Grid interface provides the single entry for users, which allow them to access all kinds of resources transparently [8]. They don't need to know who or how the services are provided. Software engineers can get a more rapid development through unified interface.

\section{3) Flexibility and Friendly Management Mechanism}

The dynamic and self-similarity of Grid ensures new users can join the Grid system easily [4]. Users can readily increase hardware facilities, and add new functions or features to systems according to their own needs instead of changing the whole system framework.

\section{The Model And Design of Digital Campus Based on OGSA}

\subsection{The model of OGSA-based digital campus}

Ordinary digital campus consists of hardware, digital library, campus self-help MMS subsystem, comprehensive management system (teaching, office, and personnel), campus card subsystem, high performance computing systems, long-distance multimedia teaching subsystems, computer network and communication equipment[9]. Fig. 2 shows the structure model of digital campus.

On the basis of campus network, we utilize OGSA to establish a model of the digital campus, which can efficiently integrate all available computer resources and solve the problems existing in current campus networks. It is shown in Fig. 3. 


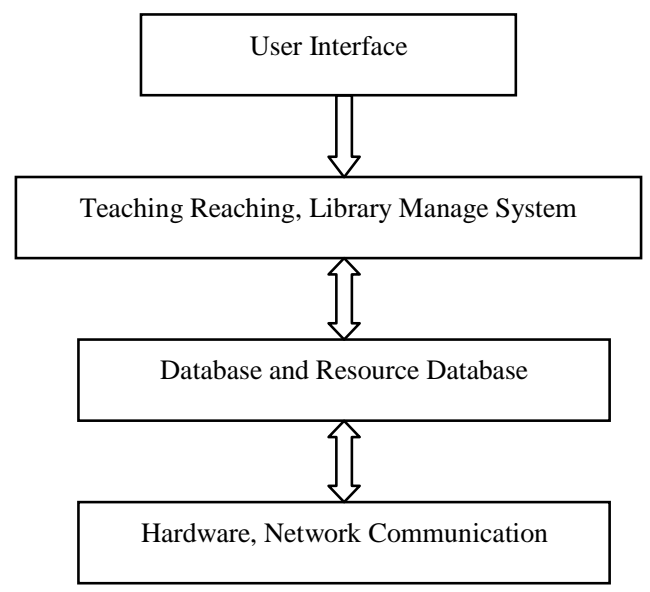

Fig. 2. The structure model of digital campus network

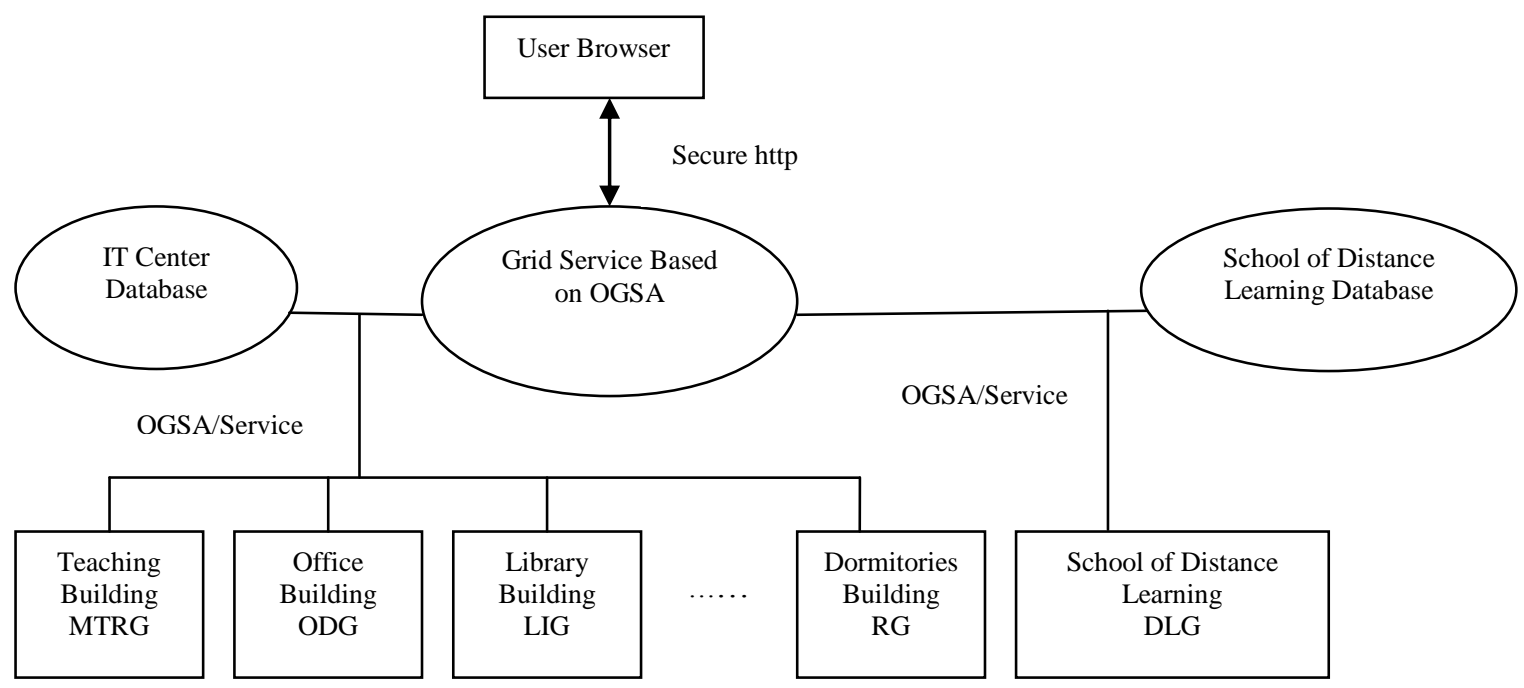

Fig. 3. The model of digital campus based on OGSA

\subsection{The design of OGSA-based digital campus}

The above model consists of different types of Grids, for example, Multimedia Teaching Resource Grid (MTRG) in teaching buildings, Office Data Grid (ODG) in office buildings, Distance Learning Grid (DLG) in school of distance learning, Resource Grid (RG) in dormitories. 


\section{1) Model and Design of DLG}

DLG consists of three parts, as is shown in Fig. 4. Date Grid(DG), computing Grid(CG), and Information Grid(IG).DG provides database for teaching materials, such as courseware, video, exercises; CG provides assistance for online homework, live video and audio; IG provides information on different subjects such as experimental date and processes etc. Establishing DLG according to OGSA can provide more numerous and efficient forms of distance education.

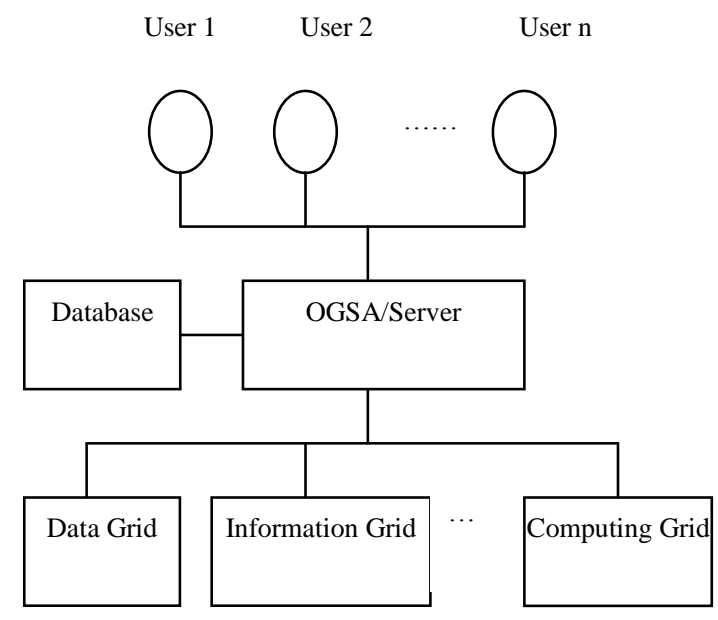

Fig. 4. Model of DLG

\section{2) Model and Design of MTRG}

The design principal of MTRG is to provide huge storage space and multimedia teaching resources for users, which are stored in different locations. In all teaching buildings with MTRG, we can use these teaching resources integrated and optimized by MTRG, such as videos and courseware.

\section{3) Model and Design of ODG}

The goal of establishing ODO is to alleviate the burden of servers in the IT center and facilitate resource sharing exchanges and the cooperation of different teaching and management departments [9]. When the lowburden PCs' CPUs in offices are idle, they can participate in tasks running on servers in the IT center, and also share part of their storage capacity and other resources.

\section{3. the superiority of establishing OGSA-based digital campus}

Establishing OGSA-based digital campus integrates all kinds of educational resources and resolves the problems existing in current campus network, especially effectively eliminate the deficiencies in multimedia teaching and distance teaching. Some universities in china have applied grid technology to their campus and benefited from it a lot, Table 1 shows the differences between network-based digital and OGSA-based digital campus, form which we can see the great comparative advantages and superiorities of establishing OGSA-based digital campus. 
Table 1. Differences between network-based digital campus and OGSA-based digital campus

\begin{tabular}{lll}
\hline & \multicolumn{1}{c}{ Network-based } & OGSA-based \\
\hline Data distribution & centralized & distributed \\
Storage capacity & comparative small & very large \\
Computing capacity & in a low and limited level & in a very high level \\
Resources sharing & partly & powerful, effective \\
Relying on servers & in a high degree & in a lower degree \\
expansibility & depending on burden of servers & a very high flexibility \\
\hline
\end{tabular}

\section{Conclusion}

With the continuous development and improvement of Grid technology, campus Grid will gradually become the indispensable infrastructure in digital campus construction, and play an irreplaceable role in teaching, scientific research and management of universities. The application of OGSA-based digital campus can fully realize resource sharing and collaboration of personalized services, and resolve the problems existing in current campus network environments. As an important foundation of the future digital campus, establishing campus Grid is imperative. This engineering is of great significance in education development and innovation, and will be another milestone in applying new technology to the education field.

\section{References}

[1] Ian Foster,Carl Kesselman,The Grid blueprint for a new computing infrastructure(second edition), China Machine Press, 2005.5.4

[2] http://en.wikipedia.org/wiki/Grid_computing\#Definitions

[3] Zhang Shunli,Yin Qingshuang, "Research of network teaching platform based grid", unpublished.

[4] Gao Hongqing, Wang Guoan, "Research of digital campus application based on grid", Journal of Henan University(Natural Science), 2005(9),vol.35,2005.9(3),pp:89-100(in Chinese)

[5] Guo $\mathrm{Yu}$, "On the Grid in the field of education and analysis of the application", Computer Knowledge and Technology. 2008, vol. 2(18), pp. 1684-1688(in Chinese)

[6] Wang Li, Jiang Tianfa, Tianfa "Discussion and design of campus Grid style architecture", Modern Electronics Technique 2008, 31(1) (in Chinese)

[7] Wang Fei and Zheng Xiaowei, "The research and design of network teaching system based on Grid technology", Journal of Liaoning Normal University (Natural Science Edition). Vol. 32, 2009(1), pp. 6163(in Chinese)

[8] FU Guo-yu, ZHOU Min, "OGSA-based Campus Grid and Job Service" , Communications Technology,vol.42,2009(3),pp.245-246(in Chinese)

[9] Nei Ruihua,Zhang Zewen,Luo Huiqong, "Study on model of campus Grid based on OGSA", Journal of south china normal university (natural science edition) 2006(4), PP: 45-51 